\title{
Cardiovascular characteristics of chronic fatigue syndrome
}

\author{
SARA BOZZINI ${ }^{1}$, ANDREA ALBERGATI ${ }^{2,3}$, ENRICA CAPELLI $^{4}$, LORENZO LORUSSO $^{5}$, \\ CARMINE GAZZARUSO ${ }^{1,3,6}$, GABRIELE PELISSERO ${ }^{3}$ and COLOMBA FALCONE ${ }^{1,3,7}$ \\ ${ }^{1}$ Interdepartmental Center for Research in Molecular Medicine (CIRMC), University of Pavia, I-27100 Pavia; \\ ${ }^{2}$ Department of Neurology, Istituti Clinici di Pavia e Vigevano, University Hospital, I-27100 Pavia; \\ ${ }^{3}$ IRCCS San Donato Hospital, San Donato Milanese, I-20097 Milan; ${ }^{4}$ Department of Earth and Environmental Sciences, \\ University of Pavia, I-27100 Pavia; ${ }^{5}$ Department of Neurology, Mellino Mellini Hospital, I-25082 Chiari BS; \\ ${ }^{6}$ Internal Medicine, Diabetes and Endocrine-Metabolic Diseases Unit and the Centre for Applied Clinical Research \\ (Ce.R.C.A.), Clinical Institute Beato Matteo, I-27029 Vigevano; ${ }^{7}$ Department of Cardiology, \\ Istituti Clinici di Pavia e Vigevano, University Hospital, I-27100 Pavia, Italy
}

Received January 30, 2017; Accepted September 29, 2017

DOI: 10.3892/br.2017.1024

\begin{abstract}
Patients with chronic fatigue syndrome (CFS) commonly exhibit orthostatic intolerance. Abnormal sympathetic predominance in the autonomic cardiovascular response to gravitational stimuli was previously described in numerous studies. The aim of the current study was to describe cardiological and clinical characteristics of Italian patients with CFS. All of the patients were of Caucasian ethnicity and had been referred to our center, the Cardiology Department of the University Hospital of Pavia (Pavia, Italy) with suspected CFS. A total of 44 patients with suspected CFS were included in the present study and the diagnosis was confirmed in 19 patients according to recent clinical guidelines. The characteristics at baseline of the population confirm findings from various previous reports regarding the prevalence in females with a female to male ratio of $4: 1$, the age of onset of the pathology and the presence of previous infection by the Epstein-Barr virus, cytomegalovirus and other human herpesviruses. Despite the current data indicating that the majority of the cardiological parameters investigated are not significantly different in patients with and without CFS, a significant association between the disease and low levels of blood pressure was identified. Other pilot studies revealed a higher prevalence of hypotension and orthostatic intolerance in patients with CFS. Furthermore, many of the CFS symptoms, including
\end{abstract}

Correspondence to: Professor Colomba Falcone, Department of Cardiology, Istituti Clinici di Pavia e Vigevano, University Hospital, 27 Via Parco Vecchio, I-27100 Pavia, Italy

E-mail: colomba.falcone@unipv.it

Key words: chronic fatigue syndrome, blood pressure, epidemiology, cardiological parameters, orthostatic intolerance fatigue, vertigo, decreased concentration, tremors and nausea, may be explained by hypotension.

\section{Introduction}

Chronic fatigue syndrome (CFS) is characterized by a strong and permanent fatigue of unknown cause, which limits functional capacity and produces varying degrees of disability (1). CFS is associated, in addition to fatigue, to a wide spectrum of symptoms, including joint and muscle pain, headaches, anxiety, depressive symptoms, cognitive and sleep disorders and intolerance to physical exertion. Establishing a diagnosis has long been inhibited due to the lack of knowledge regarding the etiopathogenesis of CFS, and the difficulty in obtaining an objective and quantitative assessment of the symptoms. In recent years, due to the variety of established criteria, it has been possible to more accurately delineate CFS, which has contributed to an improved understanding of its clinical picture, and the development of potential novel therapeutic interventions $(2,3)$.

Specific investigations are required for the cardiovascular and respiratory systems. Patients with CFS commonly exhibit orthostatic intolerance, and abnormal sympathetic predominance in the autonomic cardiovascular response to gravitational stimuli was previously described (4). In addition, certain studies investigate the role of sympathetic activity in CFS patients via the analysis of heart rate variability; however, the results are inconclusive (5). Furthermore, a small left ventricular size with a low cardiac output was observed in patients with CFS, in whom orthostatic intolerance appears to be extremely common (6). In addition, in adolescents, CFS was characterized by reduced systolic blood pressure variability and sympathetic predominance of baroreflex heart rate control during orthostatic stress (7).

Thus, the aim of the present study was to evaluate the cardiological characteristics of Italian patients with suspected CFS to identify potential diagnostic parameters. An improved 
understanding of these characteristics is important for detecting potential risk factors and predictors associated with CFS, and to improve health care prevision.

\section{Materials and methods}

Patients. The patients recruited for the present study were all of Caucasian ethnicity, and were referred from March 2011 to December 2014 to the Cardiology Department of the University Hospital of Pavia (Italy) with suspected CFS. For inclusion in the current study, fatigue must have been present for at least 6 months. Any accompanying symptoms should not have preceded the onset of fatigue and be persistent or recurring for at least 6 months. The current study was in accordance with the guidelines of the Helsinki Declaration for Human Research, and was approved by the ethics committee of the University of Pavia. Subjects exhibiting a chronic disease (such as an allergy, skin disease, vascular disease or diabetes), diagnosis of fibromyalgia, irritable bowel syndrome or using drugs on a regular basis were excluded. Written informed consent was obtained from all the participants.

Data collection. The entire population underwent the following examinations: Medical history and family anamnesis, blood test, physical examination, blood pressure measurement, echocardiographic examination, basal 12-lead electrocardiogram and continuous electrocardiographic monitoring for $24 \mathrm{~h}$, stress testing cycle ergometer and blood pressure monitoring $24 \mathrm{~h}$. All patients then underwent neurological examination, which established the diagnosis of CFS.

Sociodemographic data included patient age, sex, body mass index and ethnicity. Outcomes of interest regarding onset of the illness included age of onset (age at which the patient first experienced symptoms) and the duration of illness (time in years elapsed since the onset of illness). In addition, the current study accounted for the events and exposure to infectious agents that the participants were subjected to prior to illness.

Ergometric stress test. Exercise stress testing was performed as previously described (8). Briefly, a multistage-bicycleergometer exercise stress test was performed with the patients in the supine position with an initial workload of $25 \mathrm{~W}$ and successive increments of $25 \mathrm{~W}$ every $3 \mathrm{~min}$ at a pedalling frequency of $60 \mathrm{rpm}$. A standard 12-lead electrocardiography was taken before and at the end of each stage, and at the end of the exercise. Leads V4, V5, V6 were monitored during exercise. The systolic and diastolic blood pressures were measured using a sphygmomanometer at basal conditions, at the end of each interval, at the maximum quantity of work and every 3 min in the recovery phase. During the test, the patients were interrogated continuously regarding their symptoms in terms of pain, dyspnea, vertigo and tachycardia, as well as other symptoms. The test was interrupted if one of these conditions manifested: Appearance of chest pain and/or dyspnea, exhaustion, ST segment elevation $\geq 1 \mathrm{~mm}$ without diagnostic $\mathrm{Q}$ waves, ST-segment depression $\geq 2 \mathrm{~mm}$, repetitive arrhythmias or a drop in systolic blood pressure $>10 \mathrm{mmHg}$. The test was considered positive in the presence of down sloping ST-segment depression $\geq 1 \mathrm{mV}$ below the baseline, at least
0.06-0.08 sec after the QRS complex J point in multiple leads or ST-segment elevation.

Prior to the exercise test, a pharmacological washout was performed. Calcium-channel antagonists and nitrates were halted $48 \mathrm{~h}$ before the test. $\beta$-Blockers were gradually reduced and subsequently stopped 1 week before the examination. Patients who were taking Digitalis were not included in the current study.

Heart rate variability (HRV) parameters. Twenty-four-hour Holter electrocardiogram (ECG) monitoring was performed in all patients enrolled in the study, using a three-channel tape recorder (Cardioline Click Holter Recorder; Cardioline SpA, Trento, Italy). Each tape was manually corrected for noise and ectopic beats. Mean RR intervals and the following HRV parameters were calculated as 24-h values: Standard deviation of all NN interval, square root of the mean of the sum of the square of the differences between adjacent NN intervals and number of adjacent $\mathrm{NN}$ intervals differing by $>50 \mathrm{msec}$ divided by the total number of all $\mathrm{NN}$ intervals (pNN50).

Echocardiography. Conventional echocardiography and tissue EcocolorDoppler imaging were performed. M-mode, two-dimensional and Doppler echocardiographic examinations were performed using an ultrasonographic system (MyLab $^{\text {TM } 30 G o l d ~ C a r d i o v a s c u l a r ; ~ E s a o t e ~ S p A, ~ G e n o a, ~}$ Italy) equipped with a multi-frequency transduction. The left ventricle (LV) ejection fraction was calculated from apical two- and four-chamber views using LV volumes according to the modified biplane Simpson's rule. LV enddiastolic volume and LV end-systolic volume were indexed to body surface area. Peak early (E) and late (A) filling velocities, the $\mathrm{E}: \mathrm{A}$ ratio, and $\mathrm{E}$ velocity deceleration time were measured from the LV-inflow pattern at the tips of the mitral valve at the end of expiration. Measurement of systolic pulmonary artery pressure was performed using the maximal regurgitant velocity at the tricuspid valve via continuous wave Doppler.

Statistical analysis. Continuous variables were expressed as means \pm standard deviation for normally distributed data and they were expressed as medians \pm standard deviation otherwise. Discrete data were instead analyzed using the $\chi 2$ test. $\mathrm{P}<0.05$ was considered to indicate a statistically significant difference.

\section{Results}

Clinical and demographic aspects of the study population. A total of 44 patients with suspected CFS were included in the current study ( 9 males and 35 females; mean age, $40 \pm 13$ years) as presented in Table I.

The mean age of onset of symptoms was $31 \pm 13$ years. In the current population, the onset of symptoms was fatigue/ weakness in nine patients (20\%), fever in five patients $(11 \%)$, flu-like syndrome in two patients (5\%), arthromyalgia in one patient $(2 \%)$, neurological problems in 30 patients $(68 \%)$, dyspnea in three patients (7\%) and viral infectious episodes in seven patients (16\%). In addition, one patient developed 
Table I. Clinical characteristics of the included population.

\begin{tabular}{lccc}
\hline & $\begin{array}{c}\text { Total } \\
\text { population } \\
(\mathrm{n}=44)\end{array}$ & $\begin{array}{c}\text { Patients with } \\
\text { confirmed diagnosis of CFS } \\
(\mathrm{n}=19)\end{array}$ & $\begin{array}{c}\text { Patients without } \\
\text { CFS } \\
(\mathrm{n}=25)\end{array}$ \\
\hline Males, n (\%) & $9(20 \%)$ & $3(16 \%)$ & $6(24 \%)$ \\
Age, years & $40 \pm 13$ & $40 \pm 14$ & $41 \pm 12$ \\
Body mass index & $23.7 \pm 6.2$ & $23.6 \pm 5.7$ & $23.8 \pm 6.9$ \\
Age of onset & $31 \pm 13$ & $28 \pm 13$ & $34 \pm 12$ \\
Viral infection, $\mathrm{n}(\%)$ & $18(41 \%)$ & $6(32 \%)$ & $12(48 \%)$ \\
\hline
\end{tabular}

CFS, chronic fatigue syndrome.

Table II. Biochemical characteristics of the study population.

\begin{tabular}{|c|c|c|c|c|}
\hline Variable & $\begin{array}{l}\text { Total } \\
\text { population } \\
(\mathrm{n}=44)\end{array}$ & $\begin{array}{l}\text { Patients with } \\
\text { confirmed diagnosis of CFS } \\
\qquad(\mathrm{n}=19)\end{array}$ & $\begin{array}{l}\text { Patients without } \\
\text { CFS } \\
(n=25)\end{array}$ & P-value \\
\hline Hemoglobin $(\mathrm{g} / \mathrm{dl})$ & $13.1 \pm 1.6$ & $12.9 \pm 1.2$ & $12.6 \pm 1.9$ & Ns \\
\hline Hematocrit $(\%)$ & $40 \pm 3.8 \%$ & $39.7 \pm 2.7 \%$ & $40.4 \pm 4.6$ & Ns \\
\hline Free T3 $(\mathrm{pg} / \mathrm{ml})$ & $3.6 \pm 0.6$ & $3.22 \pm 0.7$ & $3.58 \pm 0.4$ & Ns \\
\hline Free T4 (ng/dl) & $2.7 \pm 3.5$ & $2.4 \pm 1.9$ & $2.9 \pm 4.4$ & Ns \\
\hline Thyroid-stimulating hormone (microUI/ml) & $2 \pm 1.1$ & $1.96 \pm 0.9$ & $1.96 \pm 1.4$ & Ns \\
\hline Potassium $(\mathrm{mEq} / \mathrm{l})$ & $4.3 \pm 0.4$ & $4.4 \pm 0.4$ & $4.2 \pm 0.4$ & Ns \\
\hline Creatine kinase $\mathrm{Mb}(\mathrm{U} / \mathrm{l})$ & $11.5 \pm 6.8$ & $15.5 \pm 3.5$ & $7.5 \pm 3.8$ & Ns \\
\hline Creatine kinase (U/1) & $66.3 \pm 32.8$ & $61.6 \pm 16.2$ & $72.5 \pm 33.1$ & Ns \\
\hline Lactate dehydrogenase (U/l) & $283.1 \pm 90.6$ & $291.3 \pm 75.8$ & $273.2 \pm 114.6$ & Ns \\
\hline Total cholesterol (mg/dl) & $193.9 \pm 40.7$ & $181.6 \pm 24.9$ & $205.16 \pm 49.5$ & Ns \\
\hline High-density lipoprotein cholesterol (mg/dl) & $62.3 \pm 12.6$ & $57.3 \pm 13.7$ & $65.5 \pm 11.3$ & Ns \\
\hline Triglycerides (mg/dl) & $77.7 \pm 24.8$ & $79.3 \pm 24.2$ & $76.1 \pm 26.6$ & Ns \\
\hline Glycaemia (mg/dl) & $89 \pm 11.4$ & $89.4 \pm 10.6$ & $88.6 \pm 12.4$ & Ns \\
\hline Creatinine (mg/dl) & $0.8 \pm 0.2$ & $0.8 \pm 0.2$ & $0.7 \pm 0.1$ & Ns \\
\hline
\end{tabular}

CFS, chronic fatigue syndrome; Ns, not significant.

symptoms following an intestinal infectious episode and one patient developed symptoms following a surgical intervention. The following were considered as neurological problems: Amnesias, cognitive impairment, difficulty concentrating, confusion, headaches and vertigo.

Persistent symptoms included fatigue in 26 patients (59\%); unrefreshing sleep in 10 patients (23\%); pain (such as headaches) in eight patients (18\%); arthromyalgia/myalgia in 14 patients $(32 \%)$; fever in nine patients $(20 \%)$, major depression in 11 patients $(25 \%)$, and mood disorders and anxiety in 12 patients $(27 \%)$. Two patients $(4 \%)$ presented with a sore throat and five patients $(11 \%)$ exhibited lymphadenopathy. The accompanying symptoms observed in these patients underline the heterogeneity of CFS.

Viral serology testing was performed on 3-ml blood samples of all the patients at the time of recruitment and provided evidence of exposure to the Epstein-Barr virus (EBV), cytomegalovirus (CMV) and other human herpesviridae (HHV).
In the present study, the presence of past exposure to EBV was observed in 10 patients, seven patients exhibited previous CMV infection, and five patients exhibited antibodies for EBV and $\mathrm{CMV}$, indicative of previous infection with both.

Table II presents the biochemical parameters that were evaluated in the current study. No differences in the concentration of hemoglobin and hematocrit were observed. Furthermore, thyroid function appears to be similar in the two populations, as were the lipid, glucose and creatinine levels. Certain differences were observed in the muscle profiles, but the differences were not statistically significant.

In 19 of the 44 patients with suspected CFS the diagnosis was confirmed according to recent clinical guidelines (9). Of these, three were male and 16 were female (mean age, $40 \pm 14$ years). The mean age of onset of symptoms was $28 \pm 13$ years and the cause of this occurrence was viral infection $(n=6)$ and a flu-like episode $(n=5)$. The two groups were comparable regarding sex and gender. 
Table III. Cardiovascular characteristics of patients grouped according to confirmed diagnosis of CFS.

\begin{tabular}{|c|c|c|c|}
\hline Characteristic & $\begin{array}{l}\text { Patients with confirmed } \\
\text { diagnosis of CFS } \\
(n=19)\end{array}$ & $\begin{array}{l}\text { Patients without } \\
\text { CFS } \\
(n=25)\end{array}$ & P-value \\
\hline Heart rate at baseline (bpm) & $82 \pm 10$ & $77 \pm 15$ & Ns \\
\hline Ejection fraction $(\%)$ & $59 \pm 4$ & $57 \pm 16$ & Ns \\
\hline Low value of BP at 24-h monitoring (n) & $9(47 \%)$ & $4(16 \%)$ & 0.02 \\
\hline Diurnal systolic BP at 24-h monitoring (mmHg) & $109 \pm 5$ & $120 \pm 11$ & 0.03 \\
\hline Diurnal diastolic BP at 24-h monitoring ( $\mathrm{mmHg}$ ) & $69 \pm 4$ & $74 \pm 7$ & Ns \\
\hline Nocturnal systolic BP at 24-h monitoring (mmHg) & $102 \pm 7$ & $109 \pm 11$ & Ns \\
\hline Nocturnal diastolic BP at 24-h monitoring $(\mathrm{mmHg})$ & $62 \pm 5$ & $66 \pm 9$ & Ns \\
\hline Standard deviation of all NN intervals & $127.8 \pm 43.1$ & $130.6 \pm 47.4$ & Ns \\
\hline RMSSD & $45.3 \pm 37.6$ & $39.1 \pm 30.4$ & Ns \\
\hline pNN50 & $16.3 \pm 12.7$ & $18.2 \pm 13.8$ & Ns \\
\hline
\end{tabular}

CFS, chronic fatigue syndrome; BP, blood pressure; RMSSD, square root of the mean of the sum of the square of the differences between adjacent NN intervals; pNN50, number of adjacent NN intervals differing by $>50$ msec divided by the total number of all NN intervals; Ns, not significant.

Cardiological characteristics. The mean heart rate at baseline in the patients was $79 \pm 13 \mathrm{bpm}(82 \pm 10 \mathrm{bpm}$ in patients with CFS and $77 \pm 15$ bpm in patients without CFS).

The Holter ECG over $24 \mathrm{~h}$ identified the presence of rare/sporadic ventricular or supraventricular arrhythmias in 14 patients. The HRV in patients with and without confirmed diagnosis of CFS were evaluated using ECG monitoring over $24 \mathrm{~h}$. No significant differences were observed for the HRV parameters between the two subpopulations (Table III).

EcocolorDoppler in the study population excluded alteration in left ventricular segmental kinetics, four patients exhibited mitral valve prolapse and the mean ejection fraction was $58 \pm 12 \%$. With regard to the patients with confirmed diagnosis of CFS, the mean ejection fraction was $59 \pm 4 \%$; in three of these patients with confirmed diagnosis, mitral valve prolapse was observed.

The 24-h blood pressure monitoring demonstrated that nine out of 19 patients with CFS had blood pressure at the lower of the normal limits, and only one with high blood pressure. No symptomatic hypotensive episodes were recorded during the 24-h monitoring period and the nocturnal blood pressure was similar in the two subgroups.

\section{Discussion}

The profiles of the patients in the current study confirm findings from various previous reports on prevalence in Caucasian females with a female to male ratio of 4:1 (10), the age of onset of the pathology (10) and the presence of previous infection (by EBV, Candida albicans, Borrelia burgdorferi, enterovirus, CMV, other HHV, retrovirus, bornavirus, coxsackievirus B, and the hepatitis $\mathrm{C}$ virus) $(11,12)$.

A higher ratio of females is commonly reported within CFS (13-15). The prevalence in female patients is found in numerous autoimmune diseases and indicates a different regulation of gene expression between the sexes due to the action of the hormone system. However, although different studies have investigated genetic profiles associated with CFS, data thus far are scarce and different.

As previously described, CFS predominantly affects young adults aged 20-40 years, although the symptoms also exist in childhood, adolescence and in the elderly (16). In the present study, the mean age of onset for patients with confirmed diagnosis of CFS was $28 \pm 13$ years.

The largest proportion of patients (32\%) identified infectious events prior to the onset of their fatigue, such as fever, flu-like syndrome and viral infectious episodes. With regard to noninfectious events prior to illness, neurological problems (amnesias, cognitive impairment, difficulty concentrating, confusion, headaches and vertigo) or undue stress were identified to be the most common. This reflects the pathophysiology proposal for CFS; that it is a multisystem disease, which involves the interaction between the immune and central nervous system, and in which stress potentially reactivates or replicates latent viruses, such as EBV, causing the symptoms of CFS (17-21).

As a cross-sectional study, it is not possible to determine whether the weight and obesity were significant predictors for CFS or otherwise. However, only three of the CFS patients were considered overweight or obese. This is considerably less than previously reported (22) and this could be associated with debilitating and chronic nature of the disease, which leads patients to have a more sedentary lifestyle, thus significantly reducing mobility (23).

The majority of studies agree that there is an increase in heart rate in patients with CFS, at rest and under stress. In the present study, mean heart rate at baseline is not significantly different among patients with and without CFS and, during ergometric tests, no abnormalities of ventricular repolarization were observed in all of the patients. Hypothesizing a role of sympathetic/vagal disturbance during stress, the rapidity of vagal withdrawal at onset of exercise was examined in the 
present study, but no differences were identified in patients with and without CFS. In addition, the Holter ECG monitoring, which enabled analysis of HRV in the two subpopulations of patients, did not indicate any significant difference.

Despite the current data demonstrating that the majority of the cardiological parameters investigated are not significantly different in patients with and without CFS, a significant association between the disease and low blood pressure was found. Other pilot studies have identified a higher prevalence of hypotension and orthostatic intolerance in patients with CFS. Many of the CFS symptoms, including fatigue, vertigo, decreased concentration, tremors and nausea, may be explained by an autonomic dysfunction.

Previous studies have documented an alteration in autonomic cardiovascular control; this appears to be of central origin and may be a fundamental aspect of the underlying pathophysiology of CFS (24). Patients with CFS appear to have, as well as lower systolic blood pressure, exaggerated diurnal variation. The latter, in particular, appears to be inversely correlated with increasing fatigue (25).

Our study reaffirms a previous study that highlights the potential value of non-invasive autonomic parameters, such as an alteration in blood pressure, as clinical diagnostic biomarkers in this disease (26).

The current findings reveal a novel field of research for CFS, which involves the cardiovascular system, in particular blood pressure, which may trigger the symptoms of the disease itself. The hyperactivity of the immune system in response to stress that was observed in patients with this disease, together with the presence of low pressure values, may demonstrate interactions between different pathophysiological environments.

In conclusion, although the present results require confirmation in a larger population, it is apparent that CFS patients have low blood pressure. In these patients, appropriate physiotherapeutic activity ensures that constant physical activity is performed, from which the patients will also benefit from a psychological point of view.

\section{References}

1. Fukuda K, Straus SE, Hickie I, Sharpe MC, Dobbins JG and Komaroff A; International Chronic Fatigue Syndrome Study Group: The chronic fatigue syndrome: A comprehensive approach to its definition and study. Ann Intern Med 121: 953-959, 1994.

2. Brurberg KG, Fønhus MS, Larun L, Flottorp S and Malterud K: Case definitions for chronic fatigue syndrome/myalgic encephalomyelitis (CFS/ME): A systematic review. BMJ Open 4: e003973, 2014

3. Fischer DB, William AH, Strauss AC, Unger ER, Jason L, Marshall GD Jr and Dimitrakoff JD: Chronic Fatigue Syndrome: The Current Status and Future Potentials of Emerging Biomarkers. Fatigue 2: 93-109, 2014.

4. Wyller VB, Saul JP, Walløe L and Thaulow E: Sympathetic cardiovascular control during orthostatic stress and isometric exercise in adolescent chronic fatigue syndrome. Eur J Appl Physiol 102: 623-632, 2008.

5. Meeus M, Goubert D, De Backer F, Struyf F, Hermans L, Coppieters I, De Wandele I, Da Silva H and Calders P: Heart rate variability in patients with fibromyalgia and patients with chronic fatigue syndrome: A systematic review. Semin Arthritis Rheum 43: 279-287, 2013.

6. Miwa K: Cardiac dysfunction and orthostatic intolerance in patients with myalgic encephalomyelitis and a small left ventricle. Heart Vessels 30: 484-489, 2015.
7. Wyller VB, Barbieri R and Saul JP: Blood pressure variability and closed-loop baroreflex assessment in adolescent chronic fatigue syndrome during supine rest and orthostatic stress. Eur J Appl Physiol 111: 497-507, 2011.

8. Falcone C, de Servi S, Poma E, Campana C, Sciré A Montemartini C and Specchia G: Clinical significance of exercise-induced silent myocardial ischemia in patients with coronary artery disease. J Am Coll Cardiol 9: 295-299, 1987.

9. Turnbull N, Shaw EJ, Baker R, Dunsdon S, Costin N, Britton G, et al: Chronic fatigue syndrome/myalgic encephalomyelitis (or encephalopathy): diagnosis and management of chronic fatigue syndrome/myalgic encephalomyelitis (or encephalopathy) in adults and children. Royal College of General Practitioners, London, 2007.

10. Reyes M, Gary HE Jr, Dobbins JG, Randall B, Steele L, Fukuda K, Holmes GP, Connell DG, Mawle AC, Schmid DS, et al: Surveillance for chronic fatigue syndrome - four U.S. cities, September 1989 through August 1993. MMWR CDC Surveill Summ 46: 1-13, 1997.

11. Bansal AS, Bradley AS, Bishop KN, Kiani-Alikhan S and Ford B: Chronic fatigue syndrome, the immune system and viral infection. Brain Behav Immun 26: 24-31, 2012.

12. Underhill RA: Myalgic encephalomyelitis, chronic fatigue syndrome: An infectious disease. Med Hypotheses 85: 765-773, 2015.

13. Jason LA, Richman JA, Rademaker AW, Jordan KM, Plioplys AV, Taylor RR, McCready W, Huang CF and Plioplys S: A community-based study of chronic fatigue syndrome. Arch Intern Med 159: 2129-2137, 1999.

14. Lloyd AR, Hickie I, Boughton CR, Spencer O and Wakefield D: Prevalence of chronic fatigue syndrome in an Australian population. Med J Aust 153: 522-528, 1990.

15. Lawrie SM and Pelosi AJ: Chronic fatigue syndrome in the community. Prevalence and associations. Br J Psychiatry 166: 793-797, 1995

16. Johnston SC, Staines DR and Marshall-Gradisnik SM: Epidemiological characteristics of chronic fatigue syndrome/myalgic encephalomyelitis in Australian patients. Clin Epidemiol 8: 97-107, 2016.

17. Glaser R and Kiecolt-Glaser JK: Stress-associated immune modulation: Relevance to viral infections and chronic fatigue syndrome. Am J Med 105 (3A): 35S-42S, 1998.

18. Bozzini S, Boiocchi C, Carlo-Stella N, Ricevuti G and Cuccia M: The possible underworld of Chronic Fatigue Syndrome: From neurotransmitters polymorphisms to disease. J Neurol Res 2: 16-24, 2012.

19. Pasi A, Bozzini S, Carlo-Stella N, Martinetti M, Bombardieri S, De Silvestri A, Salvaneschi L and Cuccia M: Excess of activating killer cell immunoglobulin like receptors and lack of HLA-Bw4 ligands: A two edged weapon in chronic fatigue syndrome. Mol Med Rep 4: 535-540, 2011.

20. Carlo-Stella N, Bozzini S, De Silvestri A, Sbarsi I, Pizzochero C, Lorusso L, Martinetti M and Cuccia M: Molecular study of receptor for advanced glycation endproduct gene promoter and identification of specific HLA haplotypes possibly involved in chronic fatigue syndrome. Int J Immunopathol Pharmacol 22: 745-754, 2009.

21. Ortega-Hernandez OD, Cuccia M, Bozzini S, Bassi N, Moscavitch S, Diaz-Gallo LM, Blank M, Agmon-Levin N and Shoenfeld Y: Autoantibodies, polymorphisms in the serotonin pathway, and human leukocyte antigen class II alleles in chronic fatigue syndrome: Are they associated with age at onset and specific symptoms? Ann N Y Acad Sci 1173: 589-599, 2009.

22. van't Leven M, Zielhuis GA, van der Meer JW, Verbeek AL and Bleijenberg G: Fatigue and chronic fatigue syndrome-like complaints in the general population. Eur J Public Health 20: 251-257, 2010.

23. Flores S, Brown A, Adeoye S, Jason LA and Evans M: Examining the impact of obesity on individuals with chronic fatigue syndrome. Workplace Health Saf 61: 299-307, 2013.

24. Wyller VB and Helland IB: Relationship between autonomic cardiovascular control, case definition, clinical symptoms, and functional disability in adolescent chronic fatigue syndrome: An exploratory study. Biopsychosoc Med 7: 5, 2013.

25. Newton JL, Sheth A, Shin J, Pairman J, Wilton K, Burt JA and Jones DE: Lower ambulatory blood pressure in chronic fatigue syndrome. Psychosom Med 71: 361-365, 2009.

26. Frith J,Zalewski P, Klawe JJ, Pairman J, Bitner A, Tafil-Klawe M and Newton JL: Impaired blood pressure variability in chronic fatigue syndrome - a potential biomarker. QJM 105: 831-838, 2012 . 